\title{
Concerning the motion and orientation of flux transfer events produced by component and antiparallel reconnection
}

\author{
D. G. Sibeck ${ }^{1}$ and R.-Q. Lin $^{2}$ \\ Received 12 February 2011; revised 7 April 2011; accepted 14 April 2011; published 9 July 2011.
}

[1] We employ the Cooling et al. (2001) model to predict the location, orientation, motion, and signatures of flux transfer events (FTEs) generated at the solstices and equinoxes along extended subsolar component and high-latitude antiparallel reconnection curves for typical solar wind plasma conditions and various interplanetary magnetic field (IMF) strengths and directions. In general, events generated by the two mechanisms maintain the strikingly different orientations they begin with as they move toward the terminator in opposite pairs of magnetopause quadrants. The curves along which events generated by component reconnection form bow toward the winter cusp. Events generated by antiparallel reconnection form on the equatorial magnetopause during intervals of strongly southward IMF orientation during the equinoxes, form in the winter hemisphere and only reach the dayside equatorial magnetopause during the solstices when the IMF strength is very large and the IMF points strongly southward, never reach the equatorial dayside magnetopause when the IMF has a substantial dawnward or duskward component, and never reach the equatorial flank magnetopause during intervals of northward and dawnward or duskward IMF orientation. Magnetosheath magnetic fields typically have strong components transverse to events generated by component reconnection but only weak components transverse to the axes of events generated by antiparallel reconnection. As a result, much stronger bipolar magnetic field signatures normal to the nominal magnetopause should accompany events generated by component reconnection. The results presented in this paper suggest that events generated by component reconnection predominate on the dayside equatorial and flank magnetopause for most solar wind conditions.

Citation: Sibeck, D. G., and R.-Q. Lin (2011), Concerning the motion and orientation of flux transfer events produced by component and antiparallel reconnection, J. Geophys. Res., 116, A07206, doi:10.1029/2011JA016560.

\section{Introduction}

[2] A host of observations demonstrates that reconnection dominates the solar wind's interaction with the Earth's magnetosphere. Southward IMF orientations enhance both Chapman-Ferraro currents on the dayside magnetopause and the likelihood of current-driven instabilities that initiate reconnection, which in turn facilitates the flow of solar wind mass, energy, and momentum into the magnetosphere. Consistent with this prediction, ionospheric convection, the cross-polar cap potential drop, region 1 field-aligned current strengths, substorm activity, and auroral dynamics all increase when the IMF turns southward.

[3] Reconnection can occur in the presence or absence of a guide field, be steady state or transient, and be widespread or localized. Determining which mode of reconnection predominates as a function of solar wind conditions is central to understanding the nature of the solar wind-magnetosphere interaction. The earliest and most commonly invoked models

\footnotetext{
${ }^{1}$ NASA Goddard Space Flight Center, Greenbelt, Maryland, USA.

${ }^{2}$ Carderock Division, Naval Surface Warfare Center, Bethesda, Maryland, USA.

This paper is not subject to U.S. copyright. Published in 2011 by the American Geophysical Union.
}

for component (guide field) reconnection predict its occurrence along the Chapman-Ferraro current streamline that passes through the subsolar magnetopause [Sonnerup, 1974; Gonzalez and Mozer, 1974]. More recent models predict component reconnection along lines that remain subsolar but maximize the Alfvén speed characterizing the outflow from the reconnection region [Swisdak and Drake, 2007], along the locus of points that maximize shears in the magnetic fields, resulting in curves crossing the noon meridian that sometimes lie offset from the subsolar point [Trattner et al., 2007], or along lines that lie parallel to the current streamline passing through selected locations far off the equator where magnetosheath and magnetospheric magnetic fields lie antiparallel [Fear et al., 2005]. By contrast, standard antiparallel models with no guide field restrict reconnection to the locus of points where magnetosheath and magnetospheric magnetic fields lie nearly antiparallel [Crooker, 1979]. For all but strongly southward interplanetary magnetic field (IMF) orientations, these antiparallel reconnection curves lie far removed from the subsolar point and attached to the cusps.

[4] Time-dependent reconnection generates transient events in the immediate vicinity of the magnetopause with readily identifiable signatures. Whereas steady reconnection produces boundary layers with intermixed magnetospheric and magnetosheath plasma populations [Cowley, 1982], bursty 
reconnection along a single reconnection line generates bubbles [Southwood et al., 1988; Scholer, 1988], and bursty reconnection along two or more parallel reconnection lines generates flux ropes [Lee and Fu, 1985] of interconnected magnetosheath and magnetospheric magnetic field lines containing mixtures of magnetosheath and magnetospheric plasmas.

[5] Because bubbles and flux ropes displace magnetosheath and magnetospheric magnetic field lines, their motion in response to pressure gradient and magnetic curvature forces generates characteristic magnetic field signatures in the ambient media, including transient magnetic field strength enhancements, bipolar magnetic field signatures normal to the nominal magnetopause, and rotations of the magnetic field draped over the events toward directions perpendicular to event axes [Cowley, 1982; Farrugia et al., 1987; Walthour et al., 1993]. The amplitudes of the bipolar magnetic field signatures normal to the nominal magnetopause depend strongly upon the strengths of the components of the ambient magnetosheath and magnetospheric magnetic fields perpendicular to event axes [Sibeck and Lin, 2010]. When these components are large, the fields must drape over the events, generating not only localized enhancements in the total magnetic field strength but also pronounced bipolar magnetic field signatures normal to the nominal magnetopause. When the ambient magnetic field lies nearly parallel to event axes, little to no perturbation normal to the nominal magnetopause accompanies the enhanced magnetic field strengths.

[6] Transient ( 1-5 min) events exhibiting the signatures expected for flux ropes or bubbles are common in spacecraft observations near the dayside magnetopause, where they are termed flux transfer events or FTEs [Russell and Elphic, 1978]. Stronger curvature forces in the magnetosphere than in the magnetosheath displace FTEs outward, resulting in more prominent magnetosheath than magnetospheric signatures [Ding et al., 1991]. Consistent with this prediction, statistical studies record many more magnetosheath than magnetospheric events [Kuo et al., 1995; Wang et al., 2005].

[7] Although FTEs have been observed on the dayside magnetopause for northward IMF orientations [e.g., Chandler and Avanov, 2003], they are far more common for southward IMF orientations [Rijnbeek et al., 1984; Berchem and Russell, 1984]. By contrast, FTEs on the flanks of the magnetosphere show no tendency to occur preferentially for southward IMF orientations [Kawano and Russell, 1997a]. According to one school of thought, component reconnection along a curve passing through or near the subsolar point generates FTEs on both the dayside and flank magnetopause for both northward and southward IMF orientations. However, the events are difficult to observe on the dayside magnetopause during intervals of northward IMF orientation, either because 'rereconnection' hinders their development and motion [Kawano and Russell, 1997b] or because event orientations nearly parallel to draped magnetosheath and magnetospheric magnetic field lines preclude significant bipolar magnetic field signatures normal to the nominal magnetopause [Sibeck, 2009; Sibeck and Lin, 2010]. According to the other school of thought, component reconnection generates events on the dayside during intervals of southward IMF orientation, while high-latitude reconnection generates events during intervals of northward IMF orientation [Fear et al., 2005]. Events generated in both locations reach the flanks, while only those generated by dayside component reconnection appear on the dayside magnetopause.

[8] If FTEs recur sufficiently frequently and/or are sufficiently extensive, they may dominate the solar windmagnetosphere interaction. Some in situ observations indicate that the events recur on average each 7-8 min [Rijnbeek et al., 1984], but have dimensions of only 1-2 $\mathrm{R}_{\mathrm{E}}$ perpendicular to and along the magnetopause [Russell and Elphic, 1978; Fear et al., 2008]. Unless very numerous, FTEs with these dimensions and recurrence times would not dominate the overall interaction [Cowley, 1982]. Other observations indicate generation along lines extending $\sim 3-5 \mathrm{R}_{\mathrm{E}}$ or more [e.g., Dunlop et al., 2005; Wild et al., 2005]. However, global magnetohydrodynamic (MHD) models predict events that stretch for 20 Earth radii $\left(\mathrm{R}_{\mathrm{E}}\right)$ or more across the dayside magnetopause [Fedder et al., 2002; Raeder, 2006; Kuznetsova et al., 2008, 2009]. Ground-based allsky cameras and radars provide evidence for arcs and flow bursts in the high-latitude dayside ionosphere that extend over many hours in local time during intervals of strongly southward IMF orientation [e.g., Pinnock et al., 2003]. Interpreted in terms of bursty reconnection, these observations suggest the existence of FTEs with dimensions far greater than several Earth radii along the magnetopause. Events with large dimensions occurring at the rates given above could account for 30 to $100 \%$ of the solar windmagnetosphere interaction [Lockwood et al., 1990, 1995].

[9] Consequently, statistical surveys of FTE characteristics offer an opportunity to learn more about what may be the dominant mode of the solar wind-magnetosphere interaction. In particular, they can be used to distinguish between the occurrence patterns predicted for events generated by component and antiparallel reconnection. This paper employs the Cooling et al. [2001] model for the motion of reconnected magnetic field lines at the magnetopause to describe the distinctly different predictions of the component and antiparallel reconnection models for event origin, orientation, motion, and destination as a function of IMF orientation and dipole tilt. We compare these predictions with results from previous observational studies. A statistical study of FTE motion reported by Fear et al. [2007] verified the utility of the Cooling et al. model.

\section{Component Reconnection Model Predictions for a Strong IMF and No Dipole Tilt}

[10] We begin by considering the predictions of the component reconnection model for FTE motion, orientation, and signatures during intervals of strong IMF and no dipole tilt, as recently presented and discussed in much greater detail by Sibeck and Lin [2010]. Like these authors, we assume that reconnection occurs along a curve parallel to the current streamline that passes through the subsolar point and employ the Kobel and Flückiger [1994] and Alexeev et al. [2003] models for the magnetosheath and magnetospheric magnetic fields to calculate this current streamline for different IMF orientations transverse to the Sun-Earth line. Both magnetic field models require solar wind input: we set the solar wind density and velocity to typical values of 

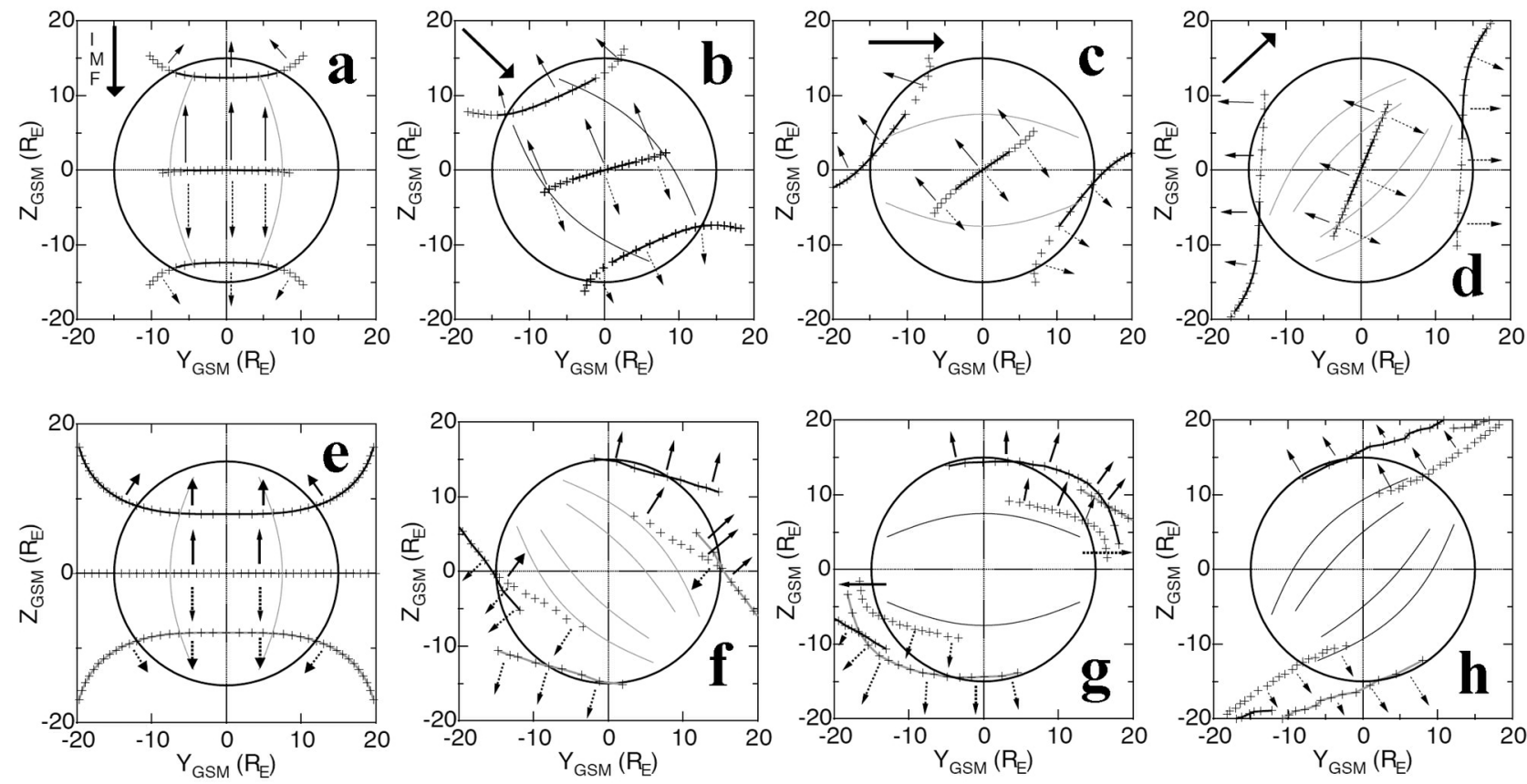

Figure 1. The motion of FTEs generated by $(\mathrm{a}-\mathrm{d})$ component and $(\mathrm{e}-\mathrm{h})$ antiparallel reconnection for southward, southward and duskward, duskward, and northward and duskward IMF orientations when there is no dipole tilt. Each panel shows a view of the dayside magnetopause from the Sun. The circles show the location of the terminator; the bold arrows in Figures 1a-1d show the IMF orientation. Crosses in Figures 1a-1d indicate the locations of points initially spaced $1 \mathrm{R}_{\mathrm{E}}$ apart on the events. Solid lines in Figures $1 \mathrm{a}-1 \mathrm{~d}$ connect points initially located within $4.5 \mathrm{R}_{\mathrm{E}}$ of the subsolar point. In Figures $1 \mathrm{e}-1 \mathrm{~h}$, no lines connect points on FTEs at the locations where they are generated; solid black lines subsequently connect points on events connected to the Northern Hemisphere, while solid gray lines subsequently connect points connected to the Southern Hemisphere. Small arrows indicate the component of event motion perpendicular to event axes at various locations. These arrows are solid where the motion of the event relative to the magnetosheath magnetic field generates bipolar out/in signatures in the magnetosheath and dashed where this motion generates in/out signatures.

$5 \mathrm{~cm}^{-3}$ and $400 \mathrm{~km} \mathrm{~s}^{-1}$, respectively. To emphasize the effects of magnetic curvature forces on FTE motion, we set the IMF strength to $10 \mathrm{nT}$, much greater than average. The magnetospheric magnetic field model also requires dipole tilt, Dst, and AL, all of which we set to zero. Consistent with results from in situ [Phan et al., 2000, 2006] and ground-based [Lockwood et al., 1990; Milan et al., 2000; Wild et al., 2003] observations, as well as numerical simulations [Fedder et al., 2002; Raeder, 2006; Kuznetsova et al., 2008, 2009], we set the initial length of the curve to $19 R_{E}$, mark off ticks each $R_{E}$, and connect those ticks initially located within $4.5 \mathrm{R}_{\mathrm{E}}$ of the subsolar point.

[11] We then employ the Cooling et al. [2001] model to determine the motion of the reconnected field lines within component reconnection events connected to the Northern and Southern Hemispheres. In this model, the locations at which the field lines cross the magnetopause move at the de Hoffman-Teller velocity, namely the sum of the local magnetosheath plasma and Alfvén velocities just outside the magnetopause. The sign of the Alfvén velocity is taken as negative for events on magnetic field lines connected to the northern ionosphere, positive for events on magnetic field lines connected to the southern ionosphere. The parameters needed to calculate these velocities can be obtained from the
Kobel-Flückiger model magnetic fields and fits to the axisymmetric gasdynamic plasma parameters predicted by Spreiter et al. [1966]. Note that neither magnetic pressures nor curvature forces have any effect upon the magnetosheath flow in the Cooling et al. model. We track the motion of each tick on the reconnection curve at $1 \mathrm{~s}$ time intervals, connecting ticks that originated at points within $4.5 \mathrm{R}_{\mathrm{E}}$ of the subsolar point, and determine event locations and shapes at subsequent times when they lie in the vicinity of the terminator at $\mathrm{X}=0$ and $\left(\mathrm{Y}^{2}+\mathrm{Z}^{2}\right)^{1 / 2}=15 \mathrm{R}_{\mathrm{E}}$.

[12] Figures 1a-1d show projections of the IMF orientation (thick solid black arrows), the terminators (large circles), draped magnetosheath magnetic field lines (faint gray curves that bow outward from the subsolar magnetopause), initial locations of events generated by the component reconnection model (curves marked by crosses that pass through the subsolar point at $Y=Z=0$ ), components of event velocities perpendicular to event motion (thin dashed and solid arrows), and event locations at times when they cross the terminators (curves marked by crosses in the vicinity of the terminators). Arrows for event motion are shown solid when that motion has a component opposite to the magnetosheath magnetic field and is expected to generate out/in bipolar magnetic field signatures normal to the 
nominal magnetopause in the magnetosheath. Arrows for event motion are shown dashed when that motion has a component parallel to the magnetosheath magnetic field and is expected to generate in/out bipolar magnetic field signatures normal to the nominal magnetopause in the magnetosheath.

[13] During periods of southward IMF (Figure 1a), the combined magnetic curvature and pressure gradient forces move events poleward. During periods of southward and duskward IMF orientation (Figure 1b), the same forces move events connected to the northern magnetosphere northward and dawnward and those connected to the southern magnetosphere southward and duskward. During periods of duskward IMF orientation (Figure 1c), they again move events either dawnward and northward or duskward and southward. Wild et al. [2007] presented a similar plot for the motion of events generated along a component reconnection line for nearly duskward IMF orientations. Finally, during periods of northward and duskward IMF orientation (Figure 1d) the combined pressure gradient and magnetic curvature forces either move events dawnward and stretch them southward or move them duskward and stretch them northward. Results for dawnward IMF orientations would be mirror images of those shown in Figures 1a-1d.

[14] Effects associated with the depletion layer [Phan et al., 1994] and stagnation line [Farrugia et al., 1998], stagnation line [Farrugia et al., 1998], and curvature forces in draped magnetosheath magnetic field lines [Lavraud et al., 2007] have not been considered here, but should enhance the speed at which events move away from subsolar component reconnection lines during intervals of strongly northward IMF orientation. The presence of a depletion layer with depressed densities and enhanced magnetic field strengths during intervals of strongly northward IMF orientation increases magnetosheath Alfven velocities. Enhanced magnetic field strengths within the depletion layer also promote the appearance of a stagnation line passing through the subsolar point and lying parallel to the draped magnetosheath magnetic field lines. Flows perpendicular to this line are enhanced. Finally, curvature forces in draped magnetosheath magnetic field lines can greatly enhance magnetosheath flow velocities just outside the magnetopause. The combined effects of the curvature forces, stagnation line, and depletion layer are to enhance the speed at which FTEs leave the dayside magnetopause during intervals of northward IMF orientation, namely the sum of the magnetosheath flow and Alfvén velocities.

[15] Inspection of Figures 1a-1d demonstrates that unless FTEs are generated along component reconnection lines that extend to the terminator and are far greater than $19 \mathrm{R}_{\mathrm{E}}$ long, they do not reach the equatorial flanks of the magnetosphere during intervals of southward IMF orientation (Figure 1a), the southern dawn or northern dusk quadrants during intervals of southward and duskward (Figure 1b) or purely duskward IMF (Figure 1c) orientation, or the polar magnetopause during intervals of northward and duskward IMF orientation (Figure 1d). While both 19 and $9 \mathrm{R}_{\mathrm{E}}$ long events reach the southern dawn and northern dusk magnetopause during intervals of northward and duskward IMF orientation, only $19 \mathrm{R}_{\mathrm{E}}$ long events reach the northern dawn and southern dusk magnetopause.

[16] The thin solid and dashed arrows in Figures 1a-1d indicate the direction (but not the magnitude) of the com- ponent of event motion perpendicular to component reconnection event axes. This is the component of event motion that causes events to move past observing spacecraft and therefore the component that can be recovered from multipoint timing studies or inferred from observations of the magnetic field orientation and the sense of bipolar magnetic field signatures normal to the nominal magnetopause. For the southward IMF case, this component lies almost in the same direction as that of the event motion. However, as the IMF rotates northward the component of event motion parallel to the event axis increases while the component perpendicular to that axis diminishes. When the IMF points due southward (Figure 1a), the component of event motion perpendicular to the event axis points poleward along the dayside equator $(Z=0)$, antisunward at high latitudes near noon (e.g., $\mathrm{Y}=0, \mathrm{Z}=10$ ), and poleward and antisunward at other locations on the high-latitude terminator (e.g., $\mathrm{Y}= \pm 8, \mathrm{Z}= \pm 12$ ). For the southward and duskward (Figure 1b), duskward (Figure 1c), and northward and duskward (Figure 1d) IMF orientations, the perpendicular component points either northward and dawnward or southward and duskward away from the putative subsolar reconnection line which stretches from southern dawn to northern dusk on the dayside magnetopause. The perpendicular components are northward and dawnward in the northern dawn and southern dusk quadrants for the southward and duskward (Figure 1b) and duskward (Figure 1c) IMF scenarios, but nearly dawnward on the dawn terminator and duskward on the dusk terminator in the northward and duskward IMF scenario (Figure 1d). There is a slight tendency for the midpoints of the events to bow toward the subsolar magnetopause, causing the perpendicular components on the ends of the events to focus inward as the events cross the terminators.

[17] Events generate outward/inward or 'direct' bipolar signatures normal to the nominal magnetopause in the magnetosheath when there is a component of event motion antiparallel to the draped magnetosheath magnetic field, but inward/outward or 'reverse' bipolar signatures when there is a component of event motion parallel to the draped magnetosheath magnetic field [Cowley, 1982]. Solid arrows in Figures 1a-1d indicate the locations north of the reconnection lines where outward/inward bipolar signatures are expected in the magnetosheath, while dashed arrows indicate the locations south of the reconnection lines where inward/ outward signatures are expected. The magnitude of the bipolar signature depends on the strength of the magnetosheath magnetic field component perpendicular to the event axes. A comparison of the faint gray traces representing draped magnetosheath magnetic field lines with the loci of points defining individual FTEs indicates substantial components at all locations shown and consequently significant bipolar magnetic field signatures.

\section{Antiparallel Reconnection Model Predictions for a Strong IMF and No Dipole Tilt}

[18] For comparison, Figures 1e-1h show predictions of the antiparallel reconnection model for FTE motion, orientation, and bipolar magnetic field signatures under the very same IMF and solar wind/magnetospheric conditions used to generate the results shown in Figures 1a-1d. Discon- 
nected crosses in Figures 1e-1h mark the initial locations of the events, crosses connected by gray curves mark the subsequent locations of events connected to the Southern Hemisphere as they cross the terminators, and crosses connected by solid black curves mark the subsequent locations of events connected to the Northern Hemisphere as they cross the terminators. Faint gray curves again illustrate draped magnetosheath magnetic field lines. Thin dashed (solid) arrows illustrate the components of event velocities perpendicular to their axes for events that move parallel (antiparallel) to the magnetosheath magnetic field and therefore generate in/out (out/in) bipolar signatures normal to the nominal magnetopause.

[19] We initiate antiparallel reconnection simultaneously along the loci of points (here disconnected crosses) where magnetosheath and magnetospheric magnetic fields lie nearly antiparallel to each other, i.e., where $\mathbf{B}_{\text {sheath }} * \mathbf{B}_{\text {sphere }} /$ $\left|\mathbf{B}_{\text {sheath }}\right|\left|\mathbf{B}_{\text {sphere }}\right|<-0.99$. During intervals of due southward IMF orientation, the antiparallel model predicts reconnection along an equatorial band stretching across all dayside longitudes whose width diminishes from cusp latitudes near local noon to the equator on the flanks. We show only an equatorial reconnection line for this case (Figure 1e). As the IMF rotates from southward and duskward (Figure 1f), through duskward (Figure 1g), to northward and duskward (Figure 1h), the model predicts reconnection curves at locations that shift from equatorial to high latitudes in the southern dawn and northern dusk sectors. Results for dawnward IMF orientations would be mirror images of those shown in Figures 1e-1h.

[20] Magnetic curvature forces cause FTEs connected to the Northern and Southern Hemisphere to move in different directions. Figures $1 \mathrm{e}-1 \mathrm{~h}$ show the locations of points on magnetic field lines rooted in the Northern (black curves connecting crosses) and Southern (gray curves connecting crosses) Hemispheres at times $200 \mathrm{~s}$ (300 s for the northward and duskward IMF orientation case) after event generation along the antiparallel reconnection curves. During periods of due southward IMF, events generated by antiparallel reconnection along lines that extend across the entire dayside magnetopause eventually reach the terminator at all latitudes. During periods of southward and duskward IMF orientation, events connected to the Northern Hemisphere move northward to the northern dusk terminator or northward and dawnward to the equatorial dawn terminator. Events connected to the Southern Hemisphere move southward and duskward to the equatorial dusk terminator or southward to the southern dawn terminator. During periods of duskward IMF orientation, events connected to the Northern Hemisphere move northward and duskward to all regions of the northern dusk terminator or dawnward to the equatorial and midlatitude southern dawn terminator. Events connected to the Southern Hemisphere move duskward to the equatorial and midlatitude northern dusk terminator or southward and dawnward to all regions of the southern dawn terminator. Wild et al. [2007] reported similar model results for FTEs generated by antiparallel reconnection during a nearly duskward IMF orientation. During periods of northward and duskward IMF orientation, events connected to the Northern Hemisphere move northward and dawnward to the high-latitude northern magnetopause or southward and dawnward to the high- latitude southern magnetopause. Events connected to the Southern Hemisphere move northward and duskward to the high-latitude northern magnetopause or southward and duskward to the high-latitude southern magnetopause.

[21] Arrows in Figures 1e-1h indicate the direction (but not the magnitude) of the component of antiparallel reconnection event velocity perpendicular to the event axes. These are the components of event motion that can be recovered from multipoint timing studies or inferred from the sense of bipolar magnetic field variations normal to the nominal magnetopause. For due southward IMF orientations (Figure 1e), these arrows point toward the nearest pole. Along the noon meridian, they point northward in the Northern Hemisphere and southward in the Southern Hemisphere. On the terminator, they point antisunward and toward the nearest pole. For southward and duskward IMF orientations (Figure 1f), the arrows point southward and dawnward at most prenoon locations but can point northward and duskward for events connected to the Northern Hemisphere in the vicinity of the dawn equator. They point northward and duskward at most postnoon locations but can point southward and dawnward for events connected to the Southern Hemisphere near the dusk equator. As the events cross the terminators, most arrows exhibit strong antisunward components. For duskward IMF orientations (Figure 1g), the arrows point southward and/or dawnward in the prenoon southern quadrant and northward and/or duskward in the postnoon northern quadrant, but northward and slightly dawnward at high latitudes in the northern dawn and southward and slightly duskward at high latitudes in the southern dusk quadrants. As the events cross the terminators, most arrows point strongly antisunward. For northward and duskward IMF orientation (Figure 1h), the arrows point northward and dawnward at northern latitudes, southward and duskward at southern latitudes. As the events cross the high latitude magnetopause, arrows point strongly antisunward and dawnward (duskward) on the northern (southern) magnetopause.

[22] Solid arrows in Figures $1 \mathrm{e}-1 \mathrm{~h}$ indicate locations where the components of event motion and the magnetosheath magnetic field perpendicular to the event axes have opposite senses and spacecraft in the magnetosheath should observe outward/inward bipolar signatures normal to the magnetopause. Dashed arrows indicate locations where these components have similar senses and spacecraft in the magnetosheath should observe inward/outward bipolar signatures. For southward IMF orientations (Figure 1e), the model predicts out/in signatures north of the equator and in/ out signatures south of the equator. For southward and duskward IMF orientations (Figure 1f), the model predicts a mixture of bipolar signatures at low latitudes, out/in signatures at high latitudes in the northern dusk quadrant, and in/out signatures in the southern dawn quadrant. For duskward IMF orientations (Figure 1g), the model predicts a transition from in/out signatures at low latitudes to out/in signatures at midlatitudes and high latitudes in the northern dusk quadrant, and a transition from out/in signatures at low latitudes to in/out signatures at midlatitudes and high latitudes in the southern dawn quadrant. For northward and duskward IMF orientations (Figure 1h), the model simply predicts out/in signatures at high northern latitudes, and in/ out signatures at high southern latitudes. Note however, that 

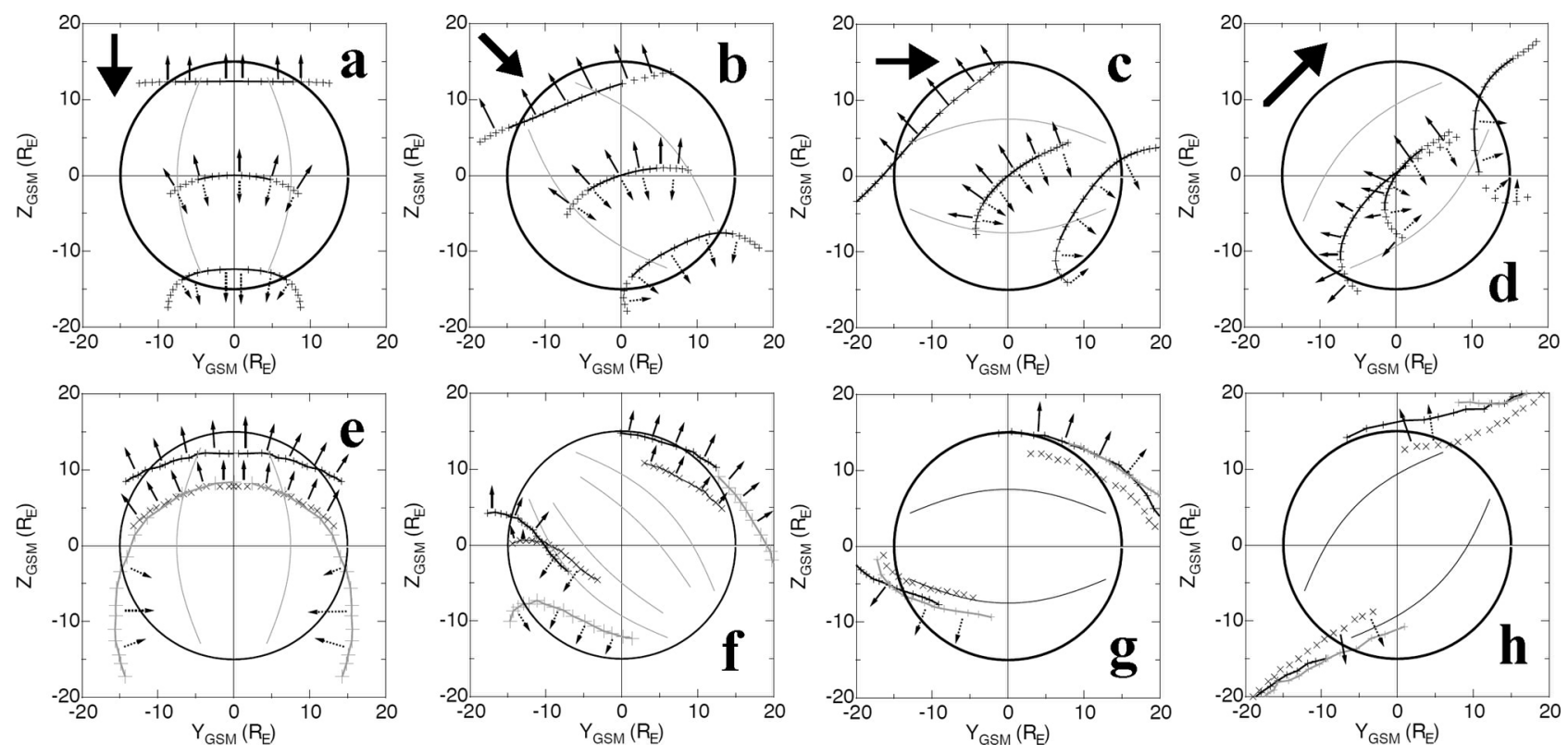

Figure 2. The motion of FTEs generated by $(\mathrm{a}-\mathrm{d})$ component and $(\mathrm{e}-\mathrm{h})$ antiparallel reconnection for southward, southward and duskward, duskward, and northward and duskward IMF orientations when the southern magnetic pole is tilted sunward. Each panel shows a view of the dayside magnetopause from the Sun. The circles show the location of the terminator; the bold arrows in Figures 2a-2d show the IMF orientation. Crosses in Figures $2 a-2 d$ indicate the locations of points initially spaced $1 R_{E}$ apart on the events. Solid lines in Figures $2 \mathrm{a}-2 \mathrm{~d}$ connect points initially located within $4.5 \mathrm{R}_{\mathrm{E}}$ of the subsolar point. In Figures 2e-2h, no lines connect points on FTEs at the locations where they are generated; solid black lines subsequently connect points on events connected to the Northern Hemisphere, while solid gray lines subsequently connect points connected to the Southern Hemisphere. Small arrows indicate the component of event motion perpendicular to event axes at various locations. These arrows are solid where the motion of the event relative to the magnetosheath magnetic field generates bipolar out/in signatures in the magnetosheath and dashed where this motion generates in/out signatures.

with the exception of the due southward IMF case shown in Figure 1e, the axes of FTEs generated by antiparallel reconnection generally lie nearly parallel to the draped magnetosheath magnetic field lines. Both the axes and magnetic field lines tilt from northern dawn to southern dusk in Figure 1f, run nearly east-west in Figure 1g, and tilt from southern dawn to northern dusk in Figure $1 \mathrm{~h}$. Since the draped field lines lie nearly parallel to event axes, the events generate only weak bipolar signatures normal to the nominal magnetopause in the magnetosheath for all but strongly southward IMF orientations.

\section{Component and Antiparallel Reconnection in the Presence of a Strong Dipole Tilt}

[23] In the presence of a dipole tilt, Chapman-Ferraro current streamlines passing through the subsolar point curl around the nearest cusp. Consequently, the projection of the component reconnection curve into the $\mathrm{Y}-\mathrm{Z}$ plane is not a straight line. Figures $2 \mathrm{a}-2 \mathrm{~d}$ present the curves along which subsolar reconnection occurs, velocity components perpendicular to event axes, the locations and orientations of events crossing the terminator, and the sense of the predicted bipolar magnetic field signatures in the magnetosheath for a $34^{\circ}$ antisunward dipole tilt (northern winter). The format is the same as that used in Figures 1a-1d.
[24] For due southward IMF orientations (Figure 2a), component reconnection events form along a subsolar curve that bows northward. Northward moving events generate out-in bipolar magnetic field signatures in the magnetosheath and straighten up as they approach the northern terminator. Southward moving events generate in/out bipolar magnetic field signatures and become more bowed as they approach the southern terminator. During southward and duskward IMF orientations (Figure 2b), events form along a subsolar reconnection curve that bows northward and dawnward. Northward and dawnward moving events generate out/in signatures and straighten up as they approach the northern dawn terminator. Southward moving events generate in/out bipolar magnetic field signatures and become more bowed as they approach the southern dusk terminator. The same is true for events generated during periods of duskward IMF orientation (Figure 2c). For northward and duskward IMF orientations (Figure 2d), events connected to the Northern Hemisphere move southward and dawnward, generating out/in bipolar magnetic field signatures normal to the nominal magnetopause in the magnetosheath. Events connected to the Southern Hemisphere move northward and duskward, generating in/out bipolar signatures normal to the nominal magnetopause.

[25] A comparison of the predictions for component reconnection shown in Figures 1a-1d with those for a strong 
dipole tilt shown in Figures $2 \mathrm{a}-2 \mathrm{~d}$ reveals numerous similarities and a few differences. Events formed by component reconnection move northward and southward away from equatorial curves for southward IMF orientations (Figures 1a and 2a), to the northern dawn and southern dusk quadrants for southward and duskward or duskward IMF orientations (Figures 1b, 1c, 2b, and 2c). Events move to the southern dawn and northern dusk quadrants for northward and duskward IMF orientations (Figures 1d and 2d), although they may extend into the northern dawn and southern dusk quadrants when there is no dipole tilt (Figure 1d). While broad regions of events generated for no dipole tilt retain the orientation of the reconnection line even as they cross the terminators (Figures 1a-1d), events generated for a strong dipole tilt (Figures 2a-2d) either straighten out (north of the reconnection line) or become more bowed (south of the reconnection line). Nevertheless, the velocity components perpendicular to event axes point either northward or southward away from equatorial reconnection lines for southward IMF orientations (Figures 1a and 2a), generally northward and dawnward or southward and duskward for southward and duskward and duskward IMF orientations (Figures 1b, 1c, 2b, and 2c). For northward and duskward IMF orientations, the velocity components for no dipole tilt point dawnward or northward and dawnward prior to local noon, duskward or southward and duskward after local noon (Figure 1d). The same is true at most locations for the case with a dipole tilt, but some vectors at high latitudes prior to local noon point southward and dawnward, while some at low latitudes after local noon point northward and duskward (Figure 2d).

[26] Now consider the results for antiparallel reconnection in the presence of a strong dipole tilt, as illustrated in Figures $2 \mathrm{e}-2 \mathrm{~h}$. For due southward IMF orientations (Figure 2e), antiparallel reconnection occurs along a curve displaced and bowed toward the winter (in this case northern) pole. Events connected to the Northern Hemisphere move northward in response to northward pressure gradients and magnetic curvature forces. For the $10 \mathrm{nT}$ IMF strength chosen in this simulation, northward pressure gradients and southward magnetic curvature forces are nearly precisely balanced, resulting in events that first stretch dawnward and duskward then southward. Had IMF strengths been weaker, pressure gradients would have moved events connected to the Southern Hemisphere northward into the winter hemisphere and no events would have been seen on the dayside equator. Had IMF strengths been greater, magnetic curvature forces would have pulled events connected to the Southern Hemisphere southward across the equatorial magnetopause. The northward moving events generate out/ in bipolar signatures normal to the nominal magnetopause. The southward moving events generate in/out bipolar magnetic fields normal to the nominal magnetopause near the terminator.

[27] For southward and duskward IMF orientations (Figure 2f), events generated by antiparallel reconnection form along curves tilted from northern dawn to southern dusk in the southern dawn and northern dusk quadrants. In the northern dusk quadrant, events connected to both the northern and Southern Hemispheres move northward and duskward, generating outward/inward bipolar signatures normal to the nominal magnetopause. In the southern dawn quadrant, events connected to the Southern Hemisphere and the southern portion of events connected to the Northern Hemisphere move southward and dawnward, generating in/ out bipolar signatures normal to the nominal magnetopause. The northern portion of events connected to the Northern Hemisphere moves northward, generating out/in bipolar signatures normal to the nominal magnetopause.

[28] For duskward IMF orientations (Figure 2g), events again form along curves running from northern dawn to southern dusk in the southern dawn and northern dusk quadrants. Events connected to both the Northern and Southern Hemisphere move southward and dawnward in the southern dawn quadrant and northward and duskward in the northern dusk quadrant. Event axes lie nearly parallel to the draped magnetosheath magnetic field lines (gray curves), and little to no bipolar magnetic field signatures normal to the nominal magnetopause are expected.

[29] For northward and duskward IMF orientations (Figure 2h), overdraping may occur [Crooker, 1992]. The magnetosheath portion of magnetic field lines reconnected poleward of the summer cusp may drape over the winter cusp. Since there is no dynamic connection between the processes occurring poleward of the cusps, overdraping does not affect the generation or evolution of the events that form in either hemisphere. These events form along curves that run from southern dawn to northern dusk in the very high latitude southern dawn and northern dusk quadrants. Events connected to the Northern Hemisphere move southward and dawnward in the southern dawn hemisphere and northward and dawnward in the northern dusk hemisphere. Events connected to the Southern Hemisphere move southward and duskward in the southern dawn hemisphere and northward and duskward in the northern dusk hemisphere. Because event axes lie nearly parallel to the draped magnetosheath magnetic field lines (gray curves), little to no bipolar magnetic field signatures normal to the nominal magnetopause are expected.

[30] With one significant exception, the predictions for antiparallel reconnection in the presence (Figures $2 \mathrm{e}-2 \mathrm{~h}$ ) and absence (Figures 1e-1h) of a significant dipole tilt are generally similar. In the absence of a significant dipole tilt, events simply move poleward away from the equatorial dayside magnetopause. In the presence of a significant dipole tilt, antiparallel reconnection generates events on the winter hemisphere magnetopause during intervals of strongly southward IMF orientation. Unless the IMF strength is very large, events connected to both the summer and winter hemisphere fail to cross the equator and simply move into the winter hemisphere. When the IMF strength is very large, only events moving toward the summer hemisphere cross the equatorial dayside magnetopause. Otherwise, the presence of a significant dipole tilt has little impact on the orientation, motion, signatures, or quadrants in which events generated by antiparallel reconnection occur when the IMF points southward and duskward, duskward, or northward and duskward.

\section{Component and Antiparallel Model Predictions for a Weak IMF}

[31] Until this point, all predictions have been for a strong (10 nT) IMF. Now consider the situation for a very weak 
Table 1. Predicted FTE Characteristics for Southward and Duskward IMF Orientations

\begin{tabular}{lll}
\hline \multicolumn{1}{c}{ Characteristic } & \multicolumn{1}{c}{ Component } & \multicolumn{1}{c}{ Antiparallel } \\
\hline Origin & Subsolar & Off-equatorial \\
Orientation & Southern dawn to northern dusk & Northern dawn to southern dusk \\
Motion/normals & Northward and dawnward or southward and duskward & Northward and duskward or southward and dawnward \\
Destination quadrant & Northern dawn and southern dusk & Northern dusk and southern dawn \\
\hline
\end{tabular}

IMF. When the IMF strength is weak, Chapman-Ferraro currents flow duskward across the dayside magnetopause to separate the strong northward magnetospheric magnetic fields from negligibly small magnetosheath magnetic fields. For southward, southward and duskward, and duskward IMF orientations, component reconnection therefore occurs along nearly equatorial reconnection lines in scenarios similar to those shown in Figures 1a, 1b, 2a, and 2b. Pressure gradients cause event lengths to increase, but east-west orientations remain almost unchanged as FTEs slip over the northern and southern magnetopause. Unless reconnection lines are much longer than $19 R_{E}$, the events fail to reach the dawn and dusk flanks. Northward moving events generate out/in bipolar signatures normal to the nominal magnetopause in the northern magnetosheath, while southward moving events generate in/out bipolar signatures normal to the nominal magnetopause in the southern magnetosheath.

[32] Whether or not component reconnection can occur for weakly northward IMF orientations is debatable. The original component reconnection models of Sonnerup [1974] and Gonzalez and Mozer [1974] forbid reconnection (and the formation of FTEs) under these circumstances because there is no reversal of the magnetosheath and magnetospheric magnetic field components across the nearly equatorial current streamline. If the reconnection line can assume any orientation [Cowley, 1976], then patchy sporadic component reconnection might still be possible [Nishida, 1989] along reconnection lines with large inclinations out of the ecliptic. The resulting FTEs would slip over the flanks in a manner similar to that illustrated in Figure 1d.

[33] Because the IMF strength plays no role in determining where antiparallel reconnection occurs in the model that we employ, the locations where antiparallel reconnection generates FTEs for weak IMF strengths are identical to those shown in Figures $1 \mathrm{e}-1 \mathrm{~h}$ and $2 \mathrm{e}-2 \mathrm{~h}$ for each IMF orientation and dipole tilt. In the absence of magnetic curvature forces, pressure gradient forces simply blow the points on these events radially antisunward away from the subsolar point. The events remain in the quadrants in which they form. Consequently, the model predicts poleward moving events during intervals of southward IMF orientation, events on the northern dusk and southern dawn magnetopause for northward and duskward, duskward, and southward and duskward IMF orientations, and on the northern dawn and southern dusk magnetopause for northward and dawnward, dawnward, and southward and dawnward IMF orientations. In the absence of magnetic curvature forces pulling events connected to the northern and southern magnetosphere in different directions, the range of latitudes over which events generated by antiparallel reconnection can be observed at the terminators for weak IMF strengths should be even more constrained than that for strong IMF strengths. Finally, northward moving events generate out/in bipolar signatures normal to the magnetopause in the magnetosheath, while southward moving events generate in/out bipolar signatures normal to the magnetopause in the magnetosheath.

\section{Comparison With Observations}

[34] This paper systematically described the often starkly contrasting predictions of the component and antiparallel reconnection models for FTE orientations, motion, occurrence patterns, and signatures during intervals of southward, southward and duskward, duskward, and northward and duskward IMF orientation. Consider the results for due southward IMF orientations first. Both component and antiparallel reconnection models predict event generation along equatorial curves during the equinoxes (Figures 1a and 1e). During the solstices, these curves bow toward the winter cusp and the antiparallel reconnection curve moves into the winter hemisphere (Figures 2a and 2e). Events generated by both component and antiparallel reconnection retain their east-west orientations as they move poleward to the high-latitude magnetopause, generating strong out/in bipolar signatures in the Northern Hemisphere and strong in/out bipolar signatures in the Southern Hemisphere (Figures 1a, 1e, 2a, and 2e). If magnetic field strengths are weak, events generated by antiparallel reconnection move toward the winter pole and fail to reach the geomagnetic equator during the solstices (Figure 2e). If magnetic fields are very strong, events generated by antiparallel reconnection move across the equator toward the summer hemisphere during the solstices.

[35] Table 1 contrasts the distinctly different predictions of the component and antiparallel reconnection models for intervals of southward and duskward or duskward IMF orientation. Component reconnection generates events that stretch from southern dawn to northern dusk along tilted curves that pass through the subsolar point (Figures 1b, 1c, $2 \mathrm{~b}$, and $2 \mathrm{c}$ ). Events retain these orientations as they move either northward and dawnward to the northern dawn magnetopause or southward and duskward to the southern dusk magnetopause. The northward moving events generate out/in bipolar signatures normal to the nominal magnetopause, while the southward moving events generate in/out signatures. By contrast, antiparallel reconnection generates events along split reconnection curves running from northern dawn to southern dusk in the southern dawn and northern dusk quadrants (Figures 1f, 1g, 2f, and 2g). The events move antisunward in the same quadrants and never reach the subsolar magnetopause. Because they lie nearly parallel to the draped magnetosheath magnetic field lines, events generated by antiparallel reconnection do not produce large bipolar perturbations in the component of the magnetosheath magnetic field normal to the magnetopause. The weak bipolar 
magnetic field signatures that they do produce are out/in north of the equator and in/out south of the equator.

[36] During intervals of northward and duskward IMF orientation, component reconnection generates events along greatly inclined curves running from southern dawn to northern dusk (Figures 1d and 2d). The events often reach the southern dawn and northern dusk flanks of the magnetosphere with even greater inclinations. They generate out/in signatures at dawn and in/out signatures at dusk. For northward and duskward IMF orientations, antiparallel reconnection also generates events with orientations that run from southern dawn to northern dusk along split curves in the southern dawn and northern dusk quadrants (Figures $1 \mathrm{~h}$ and $2 \mathrm{~h}$ ). These events retain their orientations as they move poleward and antisunward into the same quadrants. As their orientations are very similar to those of the draped magnetosheath magnetic field, the bipolar magnetic field perturbations that they generate in the direction normal to the nominal magnetopause are weak.

[37] Since the component reconnection model is the only model that predicts event occurrence on the subsolar magnetopause for IMF orientations other than strongly southward, and events on the subsolar magnetopause are known to be common for southward and dawnward or duskward IMF orientations [Berchem and Russell, 1984], component reconnection must be a major source of FTEs on the dayside magnetopause for these IMF orientations. The same model predicts that these events move to the flanks, therefore component reconnection must also be a major source of events on the flanks during intervals of southward and dawnward or duskward IMF orientation. FTEs are rare on the dayside magnetopause for strongly northward IMF orientations [Berchem and Russell, 1984], suggesting that (1) component reconnection ceases, and/or (2) that it only generates events with weak signatures, and/or (3) that reconnection moves to higher latitudes and becomes antiparallel under these circumstances. Consistent with the latter possibility, Sibeck et al. [2005] report the frequent occurrence of events for strongly antiparallel magnetospheric and magnetosheath magnetic fields at latitudes poleward of the cusps.

[38] FTEs are almost equally common on the equatorial magnetotail flanks for northward and southward IMF orientations [Kawano and Russell, 1997a]. Since the combined magnetic curvature and pressure gradient forces pull FTEs generated along equatorial reconnection curves poleward during periods of strongly southward IMF orientation, the presence of FTEs on the flank magnetopause during these conditions requires local reconnection, perhaps along equatorial curves that extend across the entire dayside magnetopause. While the component reconnection model routinely predicts event motion toward the equatorial magnetotail flanks for northward and duskward IMF orientations (Figures 1d and 2d), the antiparallel reconnection model does not (Figures $1 \mathrm{~h}$ and $2 \mathrm{~h}$ ) in the absence of very large magnetosheath magnetic field strengths. Furthermore, the bipolar magnetic field signatures normal to the nominal magnetopause should be weak for events generated by antiparallel reconnection since the magnetosheath magnetic field lies nearly parallel to event axes. The presence of events on the equatorial flanks during intervals of northward and dawnward or duskward IMF orientation then implies either that events generated by component reconnection on the dayside magnetopause during these intervals become more readily observable as they move toward the flanks of the magnetosphere [Sibeck and Lin, 2010] or that a nonstandard component reconnection model must be invoked, e. g. one in which component reconnection occurs along current streamlines that pass through selected off-equatorial locations where antiparallel reconnection is expected [Fear et al., 2005].

[39] The tests described in this paper can help determine the origin of events observed on the flanks of the magnetosphere. First, the component reconnection model predicts transitions between events generating out/in and in/out bipolar signatures normal to the magnetopause along tilted reconnection lines passing through the subsolar point, while the antiparallel reconnection model predicts transitions in the vicinity of the equator. Second, the component and antiparallel reconnection models predict events at the terminators in different pairs of quadrants. Third, the component and antiparallel reconnection models predict events with very different orientations for duskward and southward and duskward IMF orientations. Fourth, the bipolar magnetic field signatures normal to the magnetopause accompanying events on the flanks generated by the component reconnection model should generally be substantially larger than those accompanying events generated by the antiparallel reconnection model for southward and duskward, duskward, or northward and duskward IMF orientations.

[40] With respect to the first and second points, Kawano and Russell [1997b] presented results from a survey of FTEs at the dawn and dusk terminators of the magnetopause. They demonstrated that the transition between FTEs with northward (out/in bipolar magnetic field signatures) and southward (in/out bipolar signatures) occurs along an extended line passing through the subsolar point with the tilt expected for component reconnection. They also reported that dawnward IMF orientations favor event occurrence at northern dusk and southern dawn, while duskward IMF orientations favor event occurrence at southern dusk and northern dawn, again consistent with component reconnection model predictions.

[41] With respect to the third point, Fear et al. [2005] reported a survey of FTE velocities determined from multipoint timing studies of Cluster observations in the southern dusk quadrant during intervals of strongly northward and dawnward IMF orientation. The velocities they obtained generally pointed slightly northward and strongly duskward. Since timing studies can only provide the component of event velocities perpendicular to event axes, these results indicate events with orientations generally running from northern dawn to southern dusk. Figure 1d indicates that events generated by component reconnection have axes running from northern dusk to southern dawn during intervals of northward and duskward IMF orientation, corresponding to axes running from northern dawn to southern dusk during intervals of northward and dawnward IMF orientation. Consequently, the observed orientations are consistent with predictions of the component reconnection model.

[42] By contrast, Figure $1 \mathrm{~h}$ indicates that events generated by antiparallel reconnection have axes that run from southern dawn to northern dusk and move southward and duskward during intervals of northward and duskward IMF 
orientations. Events generated during intervals of northward and dawnward IMF orientation should therefore have axes that run from northern dawn to southern dusk and move southward and dawnward during intervals of northward and dawnward IMF orientations. While the latter events would have orientations consistent with those reported by Fear et al., they would not move toward and would not be observed on the equatorial or midlatitude dusk magnetopause. The locations where the Fear et al. events were observed are therefore inconsistent with the predictions of the standard antiparallel reconnection model. Nevertheless, as Fear et al. have shown, both the locations and the velocities of these events are consistent with a nonstandard model in which component reconnection occurs along current streamlines that pass through selected off-equatorial locations where antiparallel reconnection is expected.

\section{Conclusion}

[43] To summarize, we presented the strikingly different predictions of the component and antiparallel reconnection models with respect to the location of FTE generation, orientation, motion, and destination. The observational evidence reported to date preponderantly favors the component reconnection model as the cause of events observed on the equatorial to midlatitude dayside and flank magnetopause under most solar wind conditions. In the absence of extensive surveys of transient events on the polar magnetopause, little is known about the need to invoke antiparallel reconnection at higher latitudes. If such a need arises, there is no reason to suppose that component and antiparallel should not occur simultaneously. In fact, surveys of remotely sensed precipitating and mirroring ions suggest that this is frequently the case [Trattner et al., 2007].

[44] Acknowledgments. The research reported in this paper was funding by NASA's Heliophysics Guest Investigator Program. The authors thank Y. Wang for encouragement and numerous helpful suggestions.

[45] Masaki Fujimoto thanks the reviewers for their assistance in evaluating this paper.

\section{References}

Alexeev, I. I., E. S. Belenkaya, S. U. Bobrovnikov, and V. V. Kalegaev (2003), Modeling of the electromagnetic field in the interplanetary space and in the Earth's magnetosphere, Space Sci. Rev., 107, 7-26, doi:10.1023/A:1025542915800.

Berchem, J., and C. T. Russell (1984), Flux transfer events on the magnetopause: Spatial distribution and controlling factors, J. Geophys. Res., 89, 6689-6703, doi:10.1029/JA089iA08p06689.

Chandler, M. O., and L. A. Avanov (2003), Observations at low latitudes of magnetic merging signatures within a flux transfer event during a northward interplanetary magnetic field, J. Geophys. Res., 108(A10), 1358, doi:10.1029/2003JA009852.

Cooling, B. M. A., C. J. Owen, and S. J. Schwartz (2001), Role of the magnetosheath flow in determining the motion of open flux tubes, J. Geophys. Res., 106, 18,763-18,775, doi:10.1029/2000JA000455.

Cowley, S. W. H. (1976), Comments on the merging of nonantiparallel magnetic fields, J. Geophys. Res., 81, 3455-3458, doi:10.1029/ JA081i019p03455.

Cowley, S. W. H. (1982), The causes of convection in the Earth's magnetosphere: A review of developments during the IMS, Rev. Geophys., 20, 531-565, doi:10.1029/RG020i003p00531.

Crooker, N. U. (1979), Dayside merging and cusp geometry, J. Geophys. Res., 84, 951-959, doi:10.1029/JA084iA03p00951.

Crooker, N. U. (1992), Reverse convection, J. Geophys. Res., 97, 19,363-19,372, doi:10.1029/92JA01532.
Ding, D. Q., L. C. Lee, and Z. W. Ma (1991), Different FTE signatures generated by the bursty single $\mathrm{X}$ line reconnection and the multiple $\mathrm{X}$ line reconnection at the dayside magnetopause, J. Geophys. Res., 96, 57-66, doi:10.1029/90JA01989.

Dunlop, M. W., et al. (2005), Coordinated Cluster/Double Star observations of dayside reconnection signatures, Ann. Geophys., 23, 2867-2875, doi:10.5194/angeo-23-2867-2005.

Farrugia, C. J., R. C. Elphic, D. J. Southwood, and S. W. H. Cowley (1987), Field and flow perturbations outside the reconnected field line region in flux transfer events: Theory, Planet. Space Sci., 35, 227-240, doi:10.1016/0032-0633(87)90091-2.

Farrugia, C. J., H. K. Biernat, N. V. Erkaev, L. M. Kistler, G. Le, and C. T. Russell (1998), MHD model of magnetosheath flow: Comparison with AMPTE IRM observations on 24 October, 1985, Ann. Geophys., 16, 518-527, doi:10.1007/s00585-998-0518-7.

Fear, R. C., A. N. Fazakerley, C. J. Owen, and E. A. Lucek (2005), A survey of flux transfer events observed by Cluster during strongly northward IMF, Geophys. Res. Lett., 32, L18105, doi:10.1029/2005GL023811.

Fear, R. C., S. E. Milan, A. N. Fazakerley, C. J. Owen, T. Asikainen, M. G. G. T. Taylor, E. A. Lucek, H. Réme, I. Dandouras, and P. W. Daly (2007), Motion of flux transfer events: A test of the Cooling model, Ann. Geophys., 25, 1669-1690, doi:10.5194/angeo-25-1669-2007.

Fear, R. C., S. E. Milan, A. N. Fazakerley, E. A. Lucek, S. W. H. Cowley, and I. Dandouras (2008), The azimuthal extent of three flux transfer events, Ann. Geophys., 26, 2353-2369, doi:10.5194/angeo-26-23532008.

Fedder, J. A., S. P. Slinker, J. G. Lyon, and C. T. Russell (2002), Flux transfer events in global numerical simulations of the magnetosphere, J. Geophys. Res., 107(A5), 1048, doi:10.1029/2001JA000025.

Gonzalez, W. D., and F. S. Mozer (1974), A quantitative model for the potential resulting from reconnection with an arbitrary interplanetary magnetic field, J. Geophys. Res., 79, 4186-4194, doi:10.1029/ JA079i028p04186.

Kawano, H., and C. T. Russell (1997a), Survey of flux transfer events observed with the ISEE 1 spacecraft: Dependence on the interplanetary magnetic field, J. Geophys. Res., 102, 11,307-11,313, doi:10.1029/ 97JA00481.

Kawano, H., and C. T. Russell (1997b), Cause of postterminator flux transfer events, J. Geophys. Res., 102, 27,029-27,038, doi:10.1029/ 97JA02139.

Kobel, E., and E. O. Flückiger (1994), A model of the steady state magnetic field in the magnetosheath, J. Geophys. Res., 99, 23,617-23,622, doi:10.1029/94JA01778.

Kuo, H., C. T. Russell, and G. Le (1995), Statistical studies of flux transfer events, J. Geophys. Res., 100, 3513-3519, doi:10.1029/94JA02498.

Kuznetsova, M., M. Hesse, D. Sibeck, L. Rastätter, G. Toth, and A. Ridley (2008), Non-steady reconnection in global simulations of magnetosphere dynamics, Eos Trans. $A G U, 89(53)$, Fall Meet. Suppl., Abstract SM23C-04.

Kuznetsova, M. M., D. G. Sibeck, M. Hesse, L. Rastätter, G. Toth, and A. Ridley (2009), Cavities of weak magnetic field strength in the wake ofFTEs: Results from global magnetospheric MHD simulations, Geophys. Res. Lett., 36, L10104, doi:10.1029/2009GL037489.

Lavraud, B., J. E. Borovsky, A. J. Ridley, E. W. Pogue, M. F. Thompson, H. Reme, A. N. Fazakerley, and E. A. Lucek (2007), Strong bulk plasma acceleration in Earth's magnetosheath: A magnetic slingshot effect?, Geophys. Res. Lett., 34, L14102, doi:10.1029/2007GL030024.

Lee, L.-C., and Z.-F. Fu (1985), A theory of magnetic flux transfer at the Earth's magnetopause, Geophys. Res. Lett., 12, 105-108, doi:10.1029/ GL012i002p00105.

Lockwood, M., S. W. H. Cowley, P. E. Sandholt, and R. P. Lepping (1990), Signatures of flux transfer events and solar wind dynamic pressure changes, J. Geophys. Res., 95, 17,113-17,135, doi:10.1029/ JA095iA10p17113.

Lockwood, M., S. W. H. Cowley, M. F. Smith, R. P. Rijnbeek, and R. C. Elphic (1995), The contribution of flux transfer events to convection, Geophys. Res. Lett., 22, 1185-1188, doi:10.1029/95GL01008.

Milan, S. E., M. Lester, S. W. H. Cowley, and M. Brittnacher (2000), Convection and auroral response to a southward turning of the IMF: Polar UVI, CUTLASS, and IMAGE signatures of transient magnetic flux transfer at the magnetopause, J. Geophys. Res., 105, 15,741-15,756, doi:10.1029/2000JA900022.

Nishida, A. (1989), Can random reconnection on the magnetopause produce the low latitude boundary layer?, Geophys. Res. Lett., 16, 227-230, doi:10.1029/GL016i003p00227.

Phan, T.-D., G. Paschmann, W. Baumjohann, N. Sckopke, and H. Lühr (1994), The magnetosheath region adjacent to the dayside magnetopause: AMPTE/IRM observations, J. Geophys. Res., 99, 121-141, doi:10.1029/ 93JA02444. 
Phan, T.-D., et al. (2000), Extended magnetic reconnection at the Earth's magnetopause from detection of bi-directional jets, Nature, 404, 848-850, doi:10.1038/35009050.

Phan, T.-D., H. Hasegawa, M. Fujimoto, M. Øieroset, T. Mukai, and R. P. Lin (2006), Simultaneous Geotail and Wind observations of reconnection at the subsolar and flank magnetopause, Geophys. Res. Lett., 33, L09104, doi:10.1029/2006GL025756.

Pinnock, M., G. Chisham, I. J. Coleman, M. P. Freeman, M. Hairston, and J.-P. Villain (2003), The location and rate of dayside reconnection during an interval of southward interplanetary magnetic field, Ann. Geophys., 21, 1467-1482, doi:10.5194/angeo-21-1467-2003.

Raeder, J. (2006), Flux transfer events: 1. Generation mechanism for strong southward IMF, Ann. Geophys., 24, 381-392, doi:10.5194/angeo-24381-2006.

Rijnbeek, R. P., S. W. H. Cowley, D. J. Southwood, and C. T. Russell (1984), A survey of dayside flux transfer events observed by ISEE 1 and 2 magnetometers, J. Geophys. Res., 89, 786-800, doi:10.1029/ JA089iA02p00786.

Russell, C. T., and R. C. Elphic (1978), Initial ISEE magnetometer results: Magnetopause observations, Space Sci. Rev., 22, 681-715, doi:10.1007/ BF00212619.

Scholer, M. (1988), Magnetic flux transfer at the magnetopause based on single X line bursty reconnection, Geophys. Res. Lett., 15, 291-294, doi:10.1029/GL015i004p00291.

Sibeck, D. G. (2009), Concerning the occurrence pattern of flux transfer events on the dayside magnetopause, Ann. Geophys., 27, 895-903, doi:10.5194/angeo-27-895-2009.

Sibeck, D. G., and R.-Q. Lin (2010), Concerning the motion of flux transfer events across the dayside magnetopause, J. Geophys. Res., 115, A04209, doi:10.1029/2009JA014677.

Sibeck, D. G., G. I. Korotova, V. Petrov, V. Styazhkin, and T. J. Rosenberg (2005), Flux transfer events on the high-latitude magnetopause: Interball1 observations, Ann. Geophys., 23, 3549-3559, doi:10.5194/angeo-233549-2005.

Sonnerup, B. U. O. (1974), Magnetopause reconnection rate, J. Geophys. Res., 79, 1546-1549, doi:10.1029/JA079i010p01546.
Southwood, D. J., C. J. Farrugia, and M. A. Saunders (1988), What are flux transfer events?, Planet. Space Sci., 36, 503-508, doi:10.1016/00320633(88)90109-2.

Spreiter, J. R. A. L. Summers, and A. Y. Alksne (1966), Hydromagnetic flow around the magnetosphere, Planet. Space Sci., 14, 223-253, doi:10.1016/0032-0633(66)90124-3.

Swisdak, M., and J. F. Drake (2007), Orientation of the reconnection X-line, Geophys. Res. Lett., 34, L11106, doi:10.1029/2007GL029815.

Trattner, K. J., J. S. Mulcock, S. M. Petrinec, and S. A. Fuselier (2007), Probing the boundary between antiparallel and component reconnection during southward interplanetary magnetic field conditions, J. Geophys. Res., 112, A08210, doi:10.1029/2007JA012270.

Walthour, D. W., B. U. O. Sonnerup, G. Paschmann, H. Luehr, D. Klumpar, and T. Potemra (1993), Remote sensing of two-dimensional magnetopause structures, J. Geophys. Res., 98, 1489-1504, doi:10.1029/ 92JA02206

Wang, Y. L., et al. (2005), Initial results of high-latitude magnetopause and low-latitude flank flux transfer events from 3 years of Cluster observations, J. Geophys. Res., 110, A11221, doi:10.1029/2005JA011150.

Wild, J. A., et al. (2003), Coordinated interhemispheric SuperDARN radar observations of the ionospheric response to flux transfer events observed by the Cluster spacecraft at the high latitude magnetopause, Ann. Geophys., 21, 1807-1826, doi:10.5194/angeo-21-1807-2003.

Wild, J. A., et al. (2005), Simultaneous in-situ observations of the signatures of dayside reconnection at the high- and low-latitude magnetopause, Ann. Geophys., 23, 445-460, doi:10.5194/angeo-23-445-2005.

Wild, J. A., S. E. Milan, J. A. Davies, M. W. Dunlop, D. M. Wright, C. M Carr, A. Balogh, H. Reme, A. N. Fazakerley, and A. Marchaudon (2007), On the location of dayside magnetic reconnection during an interva of duskward oriented IMF, Ann. Geophys., 25, 219-238, doi:10.5194/ angeo-25-219-2007.

R.-Q. Lin, NSWCCCD, 9500 MacArthur Blvd., Code 55, Bethesda, MD 20817, USA.

D. G. Sibeck, NASA Goddard Space Flight Center, 8800 Greenbelt Rd., Code 674, Greenbelt, MD 20723, USA. (david.g.sibeck@nasa.gov) 